\title{
Article \\ Development and Validation of a High-Performance Liquid Chromatography Method for Quality Assessment of Oriental Medicine, Dokhwalgisaeng-Tang
}

\author{
Chang-Seob Seo *(D) and Hyeun-Kyoo Shin
}

Citation: Seo, C.-S.; Shin, H.-K Development and Validation of a High-Performance Liquid Chromatography Method for Quality Assessment of Oriental Medicine, Dokhwalgisaeng-Tang. Appl. Sci. 2021, 11, 7829. https://doi.org/ 10.3390/app11177829

Academic Editor: Natália Martins

Received: 13 August 2021

Accepted: 24 August 2021

Published: 25 August 2021

Publisher's Note: MDPI stays neutral with regard to jurisdictional claims in published maps and institutional affiliations.

Copyright: (c) 2021 by the authors. Licensee MDPI, Basel, Switzerland. This article is an open access article distributed under the terms and conditions of the Creative Commons Attribution (CC BY) license (https:// creativecommons.org/licenses/by/ $4.0 /)$.
KM Science Research Division, Korea Institute of Oriental Medicine, Daejeon 34054, Korea; hkshin@kiom.re.kr

* Correspondence: csseo0914@kiom.re.kr; Tel.: +82-42-868-9361

\begin{abstract}
Dokhwalgisaeng-tang (DHGST) is an herbal medicine formula that is frequently used in the treatment of arthritis in Korea and consists of 16 medicinal herbs. In this study, a simultaneous analysis method for quality assessment of DHGST by universal and widely used high-performance liquid chromatography was developed and validated. Twenty-four marker components were separated on a reverse-phase SunFire C18 column $(4.6 \times 250 \mathrm{~mm}$, particle size; $5 \mu \mathrm{m})$ maintained at $40{ }^{\circ} \mathrm{C}$ using a gradient elution of two mobile phase systems $(0.1 \%$ aqueous formic acid and $0.1 \%$ formic acid in acetonitrile). The developed method was validated via linearity, limit of detection, limit of quantification, recovery, and precision. Using the developed method, 24 marker components in DHGST were founded at $0.23-14.68 \mathrm{mg} / \mathrm{g}$, and this method will be used as basic data for the quality assessment of DHGST or other herbal medicine prescriptions.
\end{abstract}

Keywords: high-performance liquid chromatography; quality assessment; Dokhwalgisaeng-tang

\section{Introduction}

Herbal medicine prescriptions are generally composed of two or more herbal medicines and can be prepared in various formulations such as tang (decoction), pills, granules, and powders. Dokhwalgisaeng-tang (DHGST; alternatively, Duhuojisheng-tang in Chinese) is a typical herbal formula used in Korean medicine for the treatment of rheumatoid arthritis and neuritis [1]. Since DHGST was first recorded in the Bei Ji Qian Jin Yao Fang (备急千 金要方) written by Sun Simiao in the Tang Dynasty in China, it has also been included in Donguibogam (東醫寶鑑) written by Heo Jun in the Joseon Dynasty, a well-respected Korean oriental medicine book [1,2]. According to the Donguibogam, DHGST is composed of 16 medicinal herbs (Aralia continentalis Kitag., Angelica gigas Nakai, Paeonia lactiflora Pall., Taxillus chinensis (D.C.) Danser, Rehmannia glutinosa (Gaertn.) D.C., Cnidium officinale Mak., Panax ginseng C.A. Mey., Poria cocos Wolf, Achyranthes bidentata Blume, Eucommia ulmoides Oliv., Gentiana straminea Maxim., Asarum heterotropoides F. Schmidt, Saposhnikovia divaricate (Turcz.) Schischk., Cinnamomum cassia (L.) J. Presl, Glycyrrhiza uralensis Fisch., and Zingiber officinale Roscoe) and has been used to treat muscle cramps, bone pain, lower-back pain, and knee pain caused by liver-kidney Yin deficiency [1].

Many systematic reviews and meta-analyses have been reported for the use of DHGST in the treatment of rheumatoid arthritis, lumbar disk herniation, postmenopausal osteoporosis, and knee osteoarthritis [3-7], and research has been published by Liu et al. [8] on the effect of DHGST on stromal-cell-derived factor-1-induced inflammation and extracellular matrix degradation in human nucleus pulposus cells. It has also been reported that DHGST regulates autophagy and the P38/MAPK signaling pathway to prevent compression-induced matrix degradation and cell apoptosis in a rat model [9].

Each raw herbal medicine that makes up DHGST contains a variety of phytochemicals: namely, diterpenoids (kaurenoic acid and continentalic acid) from A. continentalis, coumarins (nodakenin, decursin, and decursinol angelate) from $A$. gigas, phenols (gallic 
acid and benzoic acid) and monoterpenoids (albiflorin and paeoniflorin) from P. lactiflora, cardiac glycosides (neritaloside and odoroside $\mathrm{H}$ ) from T. chinensis, miscellaneous (5-hydroxymethylfurfural) from $R$. alutinosa, phenylpropanoids (ferulic acid) and miscellaneous (senkyunolide A and (Z)-ligustilide) from C. officinale, triterpenoids (ginsenoside Rb1 and ginsenoside Rg1) from P. ginseng, triterpenoids (pachymic acid and polyporenic acid C) from P. cocos, steroids (ecdysterone) from A. bidentata, iridoids (geniposide and geniposidic acid) and lignans (pinoresinol diglucoside) from E. ulmoides, iridoids (gentiopicroside and loganic acid) from G. straminea, phenylpropanoids (methyleugenol and safrole) from A. heterotropoides, chromones (prim-O-glucosylcimifugin and 5-O-methylvisammioside) from S. divaricate, phenylpropanoids (cinnamic acid and cinnamaldehyde) from C. cassia, flavonoids (liquiritin and liquiritin apioside) and triterpenodis (glycyrrhizin) from G. uralensis, and phenols (6-gingerol and 6-shogaol) from Z. officinale [10-25].

Methods for the quality control of DHGST based on high-performance liquid chromatography (HPLC) have been published by Chen et al. [26] and Wang et al. [27]; however, the analysis time in the former method was very long (500 $\mathrm{min}$ ), and only four components (ferulic acid, osthole, gentiopicroside, and paeoniflorin) were detected. The method developed by Wang et al. [27] was based on only six components (chlorogenic acid, gentiopicrin, paeoniflorin, ferulic acid, glycyrrhizin, and osthole). Moreover, these studies focused on method efficacy rather than component analysis; thus, only a selection of component herbs (P. lactiflora, C. officinale, and G. uralensis) was examined, and no assay verification was performed. The development and validation of a simultaneous analysis method based on HPLC consistent quality evaluation of DHGST were therefore required and are described herein.

In this study, a simultaneous analysis method for the quality assessment of DHGST was developed and validated using standard HPLC equipment. The assay was used to monitor 24 marker components: gallic acid (1), 5-hydroxymethylfurfural (2), geniposidic acid (3), loganic acid (4), chlorogenic acid (5), gentiopicroside (6), pinoresinol diglucoside (7), albiflorin (8), prim-O-glucosylcimifugin (9), paeoniflorin (10), liquiritin apioside (11), liquiritin (12), ferulic acid (13), nodakenin (14), 5-O-methylvisammioside (15), benzoic acid (16), coumarin (17), cinnamic acid (18), cinnamaldehyde (19), glycyrrhizin (20), methyleugenol (21), safrole (22), decursin (23), and decursinol angelate (24).

\section{Materials and Methods}

\subsection{Plant Materials}

The 16 raw herbal medicines used in this experiment are listed in Table S1; the plant names were confirmed on the website "The Plant List" (http:/ / www.theplantlist.org/, accessed on 9 August 2021). These materials were purchased from Kwangmyungdag Medicinal Herbs (Ulsan, Korea). The origins of the raw herbal medicines were morphologically confirmed by Dr. Goya Choi, Korea Institute of Oriental Medicine (KIOM, Naju, Korea) according to guidelines and previous study protocols [28,29], and each material (2018-KE74-1 to 2018-KE74-16) was kept in KIOM.

\subsection{Chemicals and Reagents}

Compounds 1-24 (Figure S1) were purchased from commercial manufacturers: compounds 1, 2, 16, 17, 21, and 22 from Merck KGaA (Darmstadt, Germany); compounds 3, 8, 13, and 18-20 from Fujifilm Wako Pure Chemical Co. (Osaka, Japan); compounds 4, 7, and 9 from ChemNorm Biotech Co. (Wuhan, China); compound 5 from Acros Organics (Pittsburgh, PA, USA); compounds 6, 12, 15, and 24 from Chengdu Biopurify (Chengdu, China); compounds 10, 11, and 23 from Shanghai Sunny Biotech Co. (Shanghai, China); and compound 14 from ChemFaces (Wuhan, China). The purity of the reference compounds was confirmed to be $\geq 98.0 \%$ by HPLC analysis.

Methanol (Cat No. 9093-88, $\geq 99.9 \%$ ), acetonitrile (Cat No. 9017-88, $\geq 99.0 \%$ ), distilled water (Cat No. 4218-88), and formic acid (Cat No. 100264, 98-100\%) were HPLC-grade 
solvents or reagents and were purchased from J.T.Baker (Phillipsburg, NJ, USA) or Merck KGaA (Darmstadt, Germany).

\subsection{DHGST Sample Preparation}

DHGST powder extract was prepared according to a previously developed protocol [29]; the 16 herbal medicines were mixed in the weight ratio (w/w) shown in Table S1, then $50 \mathrm{~L}$ of distilled water was added, and the mixture was extracted at $100^{\circ} \mathrm{C}$ for $2 \mathrm{~h}$ using an electric extractor. The extract solution was freeze-dried with an LP110R freeze-dryer (IlShinBioBase, Dongducheon, Korea) to obtain $1113.6 \mathrm{~g}$ (yield 22.3\%) of a powder sample. The prepared DHGST sample was stored at $-20{ }^{\circ} \mathrm{C}$.

\subsection{HPLC Simultaneous Quantification of the 24 Marker Compounds}

Simultaneous quantification of the selected 24 marker analytes in the DHGST sample was conducted using a modification of a protocol developed in a previous study [29]. A Prominence LC-20A series (Shimadzu, Kyoto, Japan) HPLC instrument coupled with a photodiode array (PDA) detector capable of scanning the $190-800 \mathrm{~nm}$ region was used. The system was controlled and operated with LabSolution software (Ver. 5.53, SP3,Shimadzu, Kyoto, Japan). Full details of the analysis conditions are provided in Table S2.

A sample solution for simultaneous determination of the 24 marker analytes in the DHGST sample was prepared at a concentration of $10.0 \mathrm{mg} / \mathrm{mL}$ using $70 \%$ methanol, followed by ultrasonic extraction for $60 \mathrm{~min}$. A standard solution of each reference standard compound was prepared at a concentration of $1.0 \mathrm{mg} / \mathrm{mL}$ using methanol and then stored in a refrigerator. All of the prepared solutions were filtered through a $0.2-\mu \mathrm{m}$ membrane filter (Pall Life Sciences, Ann Arbor, MI, USA) before injection into the HPLC.

\subsection{System Suitability Test of the Analytical Method}

System suitability tests were conducted to evaluate the retention factor $\left(k^{\prime}\right)$, relative retention $(\alpha)$, resolution $(R s)$, number of theoretical plates $(N)$, and tailing factor $(T f)$ to ensure the adequate performance of the chromatography system for the developed method.

\subsection{Method Validation of the Developed HPLC Analytical Assay}

Validation of the analytical method developed in this study was performed with respect to linearity, limit of detection (LOD), limit of quantification (LOQ), accuracy, and precision as described in the previous studies $[29,30]$.

\section{Results and Discussion}

\subsection{Selection of Marker Components for Quality Assessment of DHGST}

As shown in Figure S2, we analyzed and compared the major components of each raw herbal medicine to select the marker compounds. All herbal medicines and components were scanned from 190 to $400 \mathrm{~nm}$ with a PDA detector during HPLC analysis using a mobile phase system of distilled-water-acetonitrile, with both phases containing $0.1 \%$ formic acid. Each marker component was confirmed by comparing its retention time and UV spectrum with those of the corresponding reference standard.

As shown in Figure S3, 40 main components in DHGST were analyzed using HPLCPDA to enable the selection of the marker components. As a result, 24 components were identified as suitable markers in the DHGST sample, and these analytes were used as marker compounds for quality control of the DHGST sample in the subsequent studies.

\subsection{Establishment of Optimal HPLC-PDA Conditions}

HPLC-PDA analytical conditions for efficient separation of the 24 selected markers were determined using a range of reverse-phase C18 columns (I. D. $4.6 \mathrm{~mm} \times$ length $250 \mathrm{~mm}$, particle size $5 \mu \mathrm{m}$ ), Waters SunFire (Milford, MA, USA), Thermo Scientific Hypersil GOLD (Waltham, MA, USA), Phenomenex Gemini (Torrance, CA, USA), and Shiseido Capcell Pak UG80 (Tokyo, Japan), acids ( $0.1 \%$ formic acid, $0.1 \%$ trifluoroacetic acid, and 
$1.0 \%$ acetic acid), and column oven temperatures $\left(30,40\right.$, and $\left.50{ }^{\circ} \mathrm{C}\right)$. As a result of comparative analysis, as shown in Table S2, a SunFire C18 reverse-phase column, $0.1 \%$ formic acid, and a column oven temperature of $40{ }^{\circ} \mathrm{C}$ were established as the optimal analysis conditions. Compounds 1-24 were eluted within $60 \mathrm{~min}$ and detected at 6.21, 8.70, 9.43, $12.26,14.79,16.63,17.42,17.86,18.75,18.96,20.90,21.30,22.04,22.52,23.36,25.64,28.45$, $32.79,36.05,42.76,47.93,53.20,55.39$, and $55.83 \mathrm{~min}$, respectively (Figure 1).
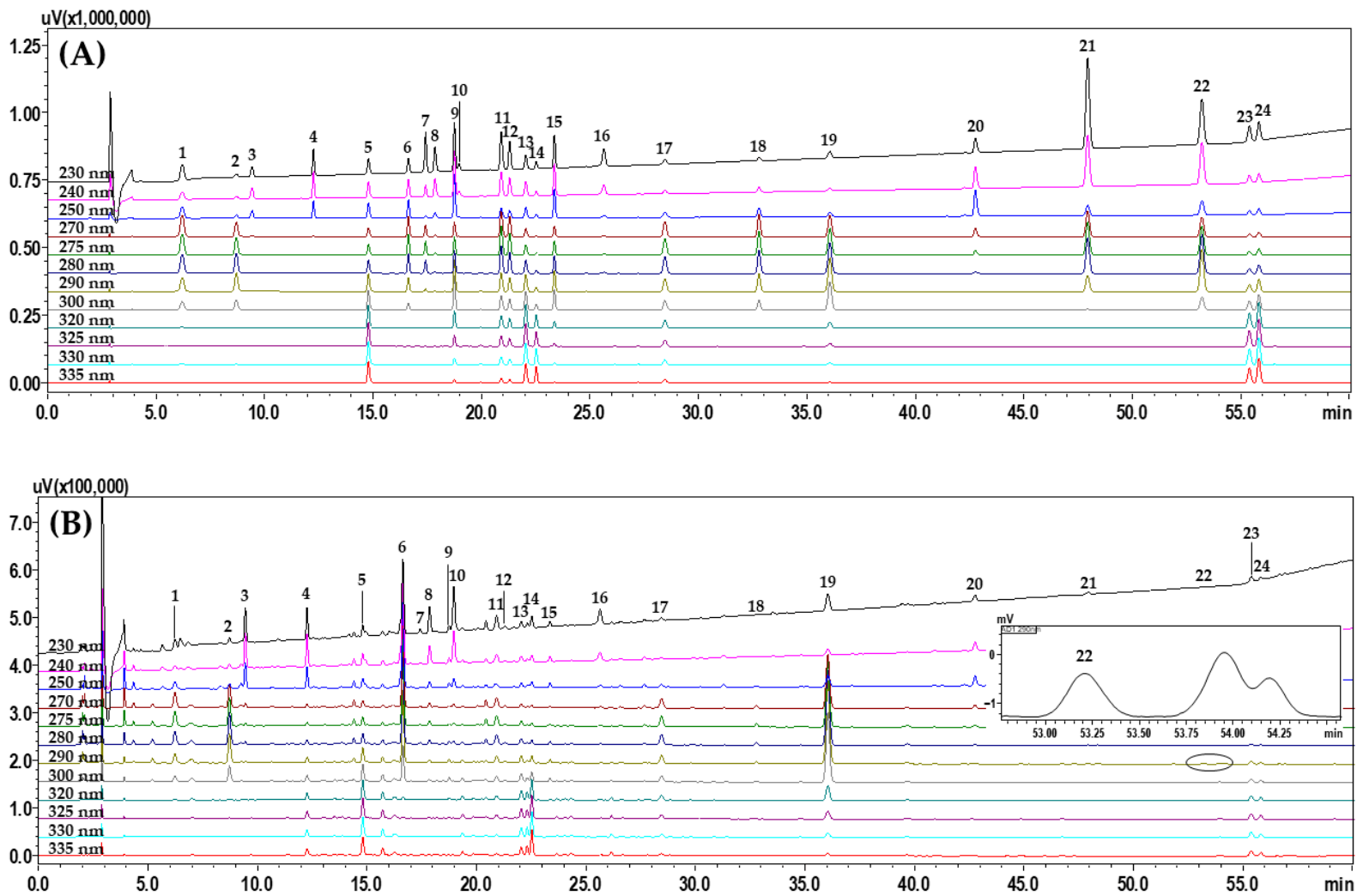

Figure 1. Representative HPLC chromatograms of (A) standard solution and (B) 70\% methanol extract of DHGST sample. Peaks correspond to gallic acid (1), 5-hydroxymethylfurfural (2), geniposidic acid (3), loganic acid (4), chlorogenic acid (5), gentiopicroside (6), pinoresinol diglucoside (7), albiflorin (8), prim-O-glucosylcimifugin (9), paeoniflorin (10), liquiritin apioside (11), liquiritin (12), ferulic acid (13), nodakenin (14), 5-O-methylvisammioside (15), benzoic acid (16), coumarin (17), cinnamic acid (18), cinnamaldehyde (19), glycyrrhizin (20), methyleugenol (21), safrole (22), decursin (23), and decursinol angelate (24).

\subsection{System Suitability and Method Validation of the Developed HPLC Analytical Method}

For efficient simultaneous analysis of markers in the DHGST sample, the suitability of the HPLC instrument was confirmed with respect to $k^{\prime}(1.15-18.38), \alpha(1.01-1.75)$, $N$ (16865-2158966), Rs (1.27-18.17), and Tf (0.92-1.20) (Table S3). The regression equations for all the calibration curves showed excellent linearity, with a coefficient of determination $\left(r^{2}\right)$ of 0.9999 to 1.0000 over the tested concentration range. The LOD and LOQ were calculated to be $0.004-0.061 \mu \mathrm{g} / \mathrm{mL}$ and $0.012-0.184 \mu \mathrm{g} / \mathrm{mL}$, respectively (Table 1). Recovery was tested by adding three levels (low, medium, and high) of each standard solution to the DHGST sample, with results in the range of $95.47-102.81 \%$ (Table 2). The repeatability of the assay with respect to retention time and peak area was measured using a DHGST sample, and the RSD (\%) was found to be $\leq 0.44 \%$ and $\leq 1.78 \%$, respectively (Tables S4 and S5). Good RSD (\%) values for intraday and interday precisions of $\leq 0.79 \%$ and $\leq 1.42 \%$, respectively, were also recorded (Table 3). Having validated the method as described, it was concluded that the current HPLC simultaneous analysis method is suitable for the quality assessment of DHGST samples. 
Table 1. Linear range, regression equation, coefficient of determination $\left(r^{2}\right)$, limit of detection (LOD), and limit of quantification (LOQ) of 24 marker analytes for quantification by HPLC $(n=3)$.

\begin{tabular}{|c|c|c|c|c|c|c|}
\hline Analyte & $\begin{array}{c}\text { Quantification } \\
\text { Wavelength } \\
\text { (nm) }\end{array}$ & $\begin{array}{l}\text { Linear Range } \\
(\mu \mathrm{g} / \mathrm{mL})\end{array}$ & $\begin{array}{l}\text { Regression Equation }^{a} \\
y=a x+b\end{array}$ & $r^{2}$ & $\operatorname{LOD}^{b}(\mu \mathrm{g} / \mathrm{mL})$ & $\operatorname{LOQ}^{c}(\mu \mathrm{g} / \mathrm{mL})$ \\
\hline 1 & 270 & $0.31-20.00$ & $y=50,316.71 x-21.01$ & 1.0000 & 0.013 & 0.040 \\
\hline 2 & 280 & $0.31-20.00$ & $y=95,555.03 x+935.74$ & 1.0000 & 0.013 & 0.039 \\
\hline 3 & 240 & $0.47-30.00$ & $y=15,452.83 x+1286.82$ & 1.0000 & 0.061 & 0.184 \\
\hline 4 & 230 & $0.31-20.00$ & $y=13,392.15 x+165.98$ & 1.0000 & 0.012 & 0.037 \\
\hline 5 & 325 & $0.31-20.00$ & $y=34,622.74 x+153.82$ & 1.0000 & 0.054 & 0.164 \\
\hline 6 & 275 & $0.47-30.00$ & $y=9752.57 x+486.57$ & 1.0000 & 0.005 & 0.017 \\
\hline 7 & 275 & $0.31-20.00$ & $y=6007.63 x+176.53$ & 1.0000 & 0.009 & 0.028 \\
\hline 8 & 230 & $0.31-20.00$ & $y=12,743.33 x-1091.63$ & 0.9999 & 0.057 & 0.173 \\
\hline 9 & 300 & $0.31-20.00$ & $y=24,286.05 x+859.92$ & 1.0000 & 0.025 & 0.074 \\
\hline 10 & 230 & $0.31-20.00$ & $y=15,281.10 x-925.73$ & 1.0000 & 0.051 & 0.155 \\
\hline 11 & 275 & $0.31-20.00$ & $y=15,640.67 x+491.83$ & 1.0000 & 0.005 & 0.015 \\
\hline 12 & 275 & $0.31-20.00$ & $y=28,890.59 x+763.74$ & 1.0000 & 0.011 & 0.032 \\
\hline 13 & 320 & $0.31-20.00$ & $y=76,887.30 x+2445.47$ & 1.0000 & 0.006 & 0.018 \\
\hline 14 & 335 & $0.31-20.00$ & $y=39,517.99 x+1130.43$ & 1.0000 & 0.008 & 0.025 \\
\hline 15 & 290 & $0.31-20.00$ & $y=24,810.62 x+623.99$ & 1.0000 & 0.031 & 0.095 \\
\hline 16 & 230 & $0.31-20.00$ & $y=66,259.50 x+1750.85$ & 1.0000 & 0.004 & 0.012 \\
\hline 17 & 275 & $0.31-20.00$ & $y=65,542.40 x+1891.13$ & 1.0000 & 0.005 & 0.014 \\
\hline 18 & 275 & $0.31-20.00$ & $y=108,376.64 x+3458.00$ & 1.0000 & 0.013 & 0.039 \\
\hline 19 & 290 & $0.31-20.00$ & $y=158,240.87 x+3787.46$ & 1.0000 & 0.005 & 0.016 \\
\hline 20 & 250 & $0.31-20.00$ & $y=8285.09 x+3641.95$ & 1.0000 & 0.058 & 0.176 \\
\hline 21 & 280 & $0.31-20.00$ & $y=14,330.97 x+305.25$ & 1.0000 & 0.006 & 0.019 \\
\hline 22 & 290 & $0.31-20.00$ & $y=22,822.38 x+385.08$ & 1.0000 & 0.023 & 0.070 \\
\hline 23 & 330 & $0.31-20.00$ & $y=50,159.85 x+796.04$ & 1.0000 & 0.016 & 0.049 \\
\hline 24 & 330 & $0.31-20.00$ & $y=33,400.18 x+668.55$ & 1.0000 & 0.013 & 0.038 \\
\hline
\end{tabular}

${ }^{\mathrm{a}} y$ and $x$ indicate the peak area and concentration of each analyte, respectively; $\mathrm{LOD}^{\mathrm{b}}=3.3 \times \sigma / S$ and $\mathrm{LOQ}^{\mathrm{c}}=10 \times \sigma / S$ (where $\sigma$ and $S$ are the standard deviation of the $y$-intercept and the slope of the calibration curve, respectively).

Table 2. Recovery (\%) of 24 marker analytes in the developed analysis method $(n=5)$.

\begin{tabular}{|c|c|c|c|c|c|}
\hline Analyte & Spiked Conc. $(\mu \mathrm{g} / \mathrm{mL})$ & Measured Conc. $(\mu \mathrm{g} / \mathrm{mL})$ & Recovery (\%) ${ }^{a}$ & SD & $\operatorname{RSD}(\%)^{b}$ \\
\hline \multirow{4}{*}{1} & 2.00 & 2.00 & 100.04 & 0.77 & 0.77 \\
\hline & 4.00 & 4.08 & 101.92 & 0.98 & 0.96 \\
\hline & 8.00 & 7.94 & 99.19 & 1.12 & 1.13 \\
\hline & 2.00 & 1.97 & 98.46 & 1.25 & 1.27 \\
\hline \multirow[t]{3}{*}{2} & 4.00 & 3.99 & 99.87 & 0.44 & 0.44 \\
\hline & 8.00 & 7.78 & 97.27 & 0.51 & 0.52 \\
\hline & 1.00 & 1.01 & 101.30 & 1.16 & 1.14 \\
\hline \multirow[t]{3}{*}{3} & 2.00 & 2.03 & 101.44 & 1.84 & 1.82 \\
\hline & 4.00 & 4.07 & 101.82 & 1.55 & 1.52 \\
\hline & 1.00 & 1.02 & 101.83 & 2.05 & 2.02 \\
\hline \multirow[t]{3}{*}{4} & 2.00 & 1.97 & 98.55 & 0.55 & 0.56 \\
\hline & 4.00 & 4.02 & 100.41 & 0.92 & 0.92 \\
\hline & 2.00 & 2.03 & 101.39 & 1.20 & 1.19 \\
\hline \multirow[t]{3}{*}{5} & 5.00 & 5.03 & 100.54 & 0.53 & 0.53 \\
\hline & 10.00 & 9.96 & 99.59 & 0.93 & 0.93 \\
\hline & 3.00 & 2.98 & 99.37 & 1.32 & 1.33 \\
\hline \multirow[t]{3}{*}{6} & 7.50 & 7.30 & 97.38 & 1.47 & 1.50 \\
\hline & 15.00 & 14.48 & 96.52 & 2.34 & 2.43 \\
\hline & 2.00 & 1.94 & 97.25 & 1.31 & 1.34 \\
\hline \multirow[t]{3}{*}{7} & 5.00 & 4.92 & 98.45 & 0.82 & 0.84 \\
\hline & 10.00 & 10.08 & 100.78 & 1.49 & 1.47 \\
\hline & 1.00 & 0.97 & 97.42 & 1.63 & 1.67 \\
\hline \multirow[t]{2}{*}{8} & 2.00 & 1.97 & 98.73 & 1.72 & 1.74 \\
\hline & 4.00 & 3.86 & 96.49 & 1.16 & 1.20 \\
\hline
\end{tabular}


Table 2. Cont.

\begin{tabular}{|c|c|c|c|c|c|}
\hline Analyte & Spiked Conc. $(\mu \mathrm{g} / \mathrm{mL})$ & Measured Conc. $(\mu \mathrm{g} / \mathrm{mL})$ & Recovery $(\%)^{a}$ & SD & RSD (\%) ${ }^{b}$ \\
\hline \multirow{4}{*}{9} & 1.00 & 1.00 & 100.20 & 2.80 & 2.79 \\
\hline & 2.00 & 2.00 & 99.77 & 0.81 & 0.81 \\
\hline & 4.00 & 4.05 & 101.34 & 0.96 & 0.95 \\
\hline & 1.00 & 0.98 & 98.30 & 1.58 & 1.61 \\
\hline \multirow[t]{3}{*}{10} & 2.00 & 2.02 & 101.24 & 2.36 & 2.34 \\
\hline & 4.00 & 3.99 & 99.78 & 0.80 & 0.80 \\
\hline & 1.00 & 0.99 & 99.17 & 0.73 & 0.74 \\
\hline \multirow[t]{3}{*}{11} & 2.00 & 1.93 & 96.42 & 0.52 & 0.54 \\
\hline & 4.00 & 4.03 & 100.75 & 1.24 & 1.23 \\
\hline & 1.00 & 1.00 & 99.75 & 0.78 & 0.78 \\
\hline \multirow[t]{3}{*}{12} & 2.00 & 2.02 & 100.75 & 1.00 & 0.99 \\
\hline & 4.00 & 4.05 & 101.22 & 0.88 & 0.87 \\
\hline & 1.00 & 1.03 & 102.81 & 0.75 & 0.73 \\
\hline \multirow[t]{3}{*}{13} & 2.00 & 1.96 & 97.79 & 0.50 & 0.51 \\
\hline & 4.00 & 3.90 & 97.55 & 0.84 & 0.86 \\
\hline & 2.00 & 2.02 & 100.97 & 1.17 & 1.16 \\
\hline \multirow[t]{3}{*}{14} & 5.00 & 5.05 & 100.95 & 1.46 & 1.44 \\
\hline & 10.00 & 10.15 & 101.53 & 0.82 & 0.81 \\
\hline & 1.00 & 0.99 & 99.32 & 2.67 & 2.69 \\
\hline \multirow[t]{3}{*}{15} & 2.00 & 1.99 & 99.27 & 1.24 & 1.25 \\
\hline & 4.00 & 4.01 & 100.20 & 1.05 & 1.05 \\
\hline & 1.00 & 0.96 & 95.92 & 0.79 & 0.83 \\
\hline \multirow[t]{3}{*}{16} & 2.00 & 1.92 & 96.04 & 0.96 & 0.99 \\
\hline & 4.00 & 3.86 & 96.57 & 1.31 & 1.35 \\
\hline & 1.00 & 0.96 & 96.49 & 0.81 & 0.84 \\
\hline \multirow[t]{3}{*}{17} & 2.00 & 1.92 & 96.03 & 0.54 & 0.56 \\
\hline & 4.00 & 3.98 & 99.43 & 0.36 & 0.36 \\
\hline & 1.00 & 0.96 & 96.28 & 0.34 & 0.36 \\
\hline \multirow[t]{3}{*}{18} & 2.00 & 1.91 & 95.68 & 0.71 & 0.75 \\
\hline & 4.00 & 3.84 & 95.90 & 0.24 & 0.25 \\
\hline & 1.00 & 1.02 & 101.96 & 0.10 & 0.10 \\
\hline \multirow[t]{3}{*}{19} & 2.00 & 1.92 & 96.01 & 0.45 & 0.47 \\
\hline & 4.00 & 3.90 & 97.55 & 0.46 & 0.47 \\
\hline & 1.00 & 1.02 & 101.91 & 0.78 & 0.77 \\
\hline \multirow[t]{3}{*}{20} & 2.00 & 2.00 & 100.05 & 0.66 & 0.66 \\
\hline & 4.00 & 4.01 & 100.35 & 1.47 & 1.47 \\
\hline & 1.00 & 0.96 & 95.68 & 0.49 & 0.51 \\
\hline \multirow[t]{3}{*}{21} & 2.00 & 1.97 & 98.62 & 0.33 & 0.34 \\
\hline & 4.00 & 3.95 & 98.70 & 0.25 & 0.26 \\
\hline & 1.00 & 0.96 & 95.77 & 1.01 & 1.05 \\
\hline \multirow[t]{3}{*}{22} & 2.00 & 1.91 & 95.47 & 0.54 & 0.56 \\
\hline & 4.00 & 3.87 & 96.83 & 0.41 & 0.42 \\
\hline & 1.00 & 0.98 & 98.10 & 1.93 & 1.97 \\
\hline \multirow[t]{3}{*}{23} & 2.00 & 1.97 & 98.32 & 0.32 & 0.32 \\
\hline & 4.00 & 4.09 & 102.16 & 0.22 & 0.21 \\
\hline & 1.00 & 0.98 & 97.75 & 0.95 & 0.97 \\
\hline \multirow[t]{2}{*}{24} & 2.00 & 1.95 & 97.69 & 0.41 & 0.42 \\
\hline & 4.00 & 4.02 & 100.52 & 0.29 & 0.29 \\
\hline
\end{tabular}

Recovery $(\%)^{\mathrm{a}}=$ measured concentration/spiked concentration $\times 100 \%$; RSD $(\%)^{\mathrm{b}}=$ standard deviation (SD) $/$ mean $\times 100 \%$. 
Table 3. Precision and accuracy of the developed analysis method using 24 markers.

\begin{tabular}{|c|c|c|c|c|c|c|c|}
\hline \multirow[b]{2}{*}{ Analyte } & \multirow[b]{2}{*}{$\begin{array}{c}\text { Conc. } \\
(\mu \mathrm{g} / \mathrm{mL})\end{array}$} & \multicolumn{3}{|c|}{ Intraday $(n=5)$} & \multicolumn{3}{|c|}{ Interday $(n=5)$} \\
\hline & & $\begin{array}{c}\text { Measured } \\
\text { Conc. } \\
(\mu \mathrm{g} / \mathrm{mL})\end{array}$ & $\begin{array}{l}\text { Precision } \\
(\text { RSD, \%) }\end{array}$ & Accuracy (\%) & $\begin{array}{c}\text { Measured } \\
\text { Conc. } \\
(\mu \mathrm{g} / \mathrm{mL})\end{array}$ & $\begin{array}{l}\text { Precision } \\
\text { (RSD, \%) }\end{array}$ & Accuracy (\%) \\
\hline \multirow{4}{*}{1} & 5.00 & 4.88 & 0.46 & 97.64 & 4.87 & 0.53 & 97.38 \\
\hline & 10.00 & 9.72 & 0.44 & 97.23 & 9.68 & 0.48 & 96.83 \\
\hline & 20.00 & 19.50 & 0.19 & 97.51 & 19.49 & 0.32 & 97.44 \\
\hline & 5.00 & 4.97 & 0.47 & 99.42 & 4.96 & 0.48 & 99.24 \\
\hline \multirow[t]{3}{*}{2} & 10.00 & 9.85 & 0.73 & 98.52 & 9.79 & 1.24 & 97.90 \\
\hline & 20.00 & 19.79 & 0.39 & 98.93 & 19.65 & 0.57 & 98.27 \\
\hline & 7.50 & 7.52 & 0.14 & 100.29 & 7.58 & 1.19 & 101.05 \\
\hline \multirow[t]{3}{*}{3} & 15.00 & 14.97 & 0.25 & 99.81 & 15.07 & 0.99 & 100.43 \\
\hline & 30.00 & 29.96 & 0.79 & 99.88 & 30.04 & 0.80 & 100.14 \\
\hline & 5.00 & 5.03 & 0.20 & 100.65 & 5.05 & 0.81 & 101.03 \\
\hline \multirow[t]{3}{*}{4} & 10.00 & 9.96 & 0.25 & 99.64 & 9.99 & 0.58 & 99.93 \\
\hline & 20.00 & 19.83 & 0.18 & 99.17 & 19.98 & 0.84 & 99.92 \\
\hline & 7.50 & 7.26 & 0.70 & 96.78 & 7.19 & 0.87 & 95.17 \\
\hline \multirow[t]{3}{*}{5} & 15.00 & 14.46 & 0.73 & 96.38 & 14.30 & 0.95 & 95.32 \\
\hline & 30.00 & 29.01 & 0.53 & 96.71 & 28.80 & 0.62 & 96.00 \\
\hline & 7.50 & 7.48 & 0.18 & 99.78 & 7.55 & 1.20 & 100.04 \\
\hline \multirow[t]{3}{*}{6} & 15.00 & 14.86 & 0.20 & 99.08 & 14.96 & 0.83 & 99.75 \\
\hline & 30.00 & 29.63 & 0.19 & 98.78 & 29.88 & 0.90 & 99.61 \\
\hline & 5.00 & 4.99 & 0.35 & 99.75 & 5.03 & 1.16 & 100.55 \\
\hline \multirow[t]{3}{*}{7} & 10.00 & 9.90 & 0.23 & 98.97 & 9.96 & 0.87 & 99.60 \\
\hline & 20.00 & 19.75 & 0.10 & 98.76 & 19.89 & 0.77 & 99.45 \\
\hline & 5.00 & 4.86 & 0.37 & 97.13 & 4.90 & 1.10 & 98.00 \\
\hline \multirow[t]{3}{*}{8} & 10.00 & 10.00 & 0.32 & 99.98 & 9.86 & 1.53 & 98.55 \\
\hline & 20.00 & 19.71 & 0.40 & 98.56 & 19.89 & 0.93 & 99.43 \\
\hline & 5.00 & 5.00 & 0.32 & 100.01 & 5.04 & 1.20 & 100.75 \\
\hline \multirow[t]{3}{*}{9} & 10.00 & 9.93 & 0.27 & 99.31 & 10.00 & 0.86 & 99.98 \\
\hline & 20.00 & 19.80 & 0.22 & 99.01 & 19.95 & 0.84 & 99.77 \\
\hline & 5.00 & 4.97 & 0.85 & 99.50 & 5.02 & 1.09 & 100.32 \\
\hline \multirow[t]{3}{*}{10} & 10.00 & 9.91 & 0.62 & 99.05 & 9.95 & 0.62 & 99.50 \\
\hline & 20.00 & 19.83 & 0.63 & 99.13 & 19.95 & 1.02 & 99.73 \\
\hline & 5.00 & 4.98 & 0.14 & 99.68 & 5.02 & 1.04 & 100.26 \\
\hline \multirow[t]{3}{*}{11} & 10.00 & 9.90 & 0.22 & 98.99 & 9.96 & 0.75 & 99.43 \\
\hline & 20.00 & 19.74 & 0.22 & 98.69 & 19.95 & 0.84 & 99.38 \\
\hline & 5.00 & 4.99 & 0.11 & 99.81 & 5.03 & 1.12 & 100.50 \\
\hline \multirow[t]{3}{*}{12} & 10.00 & 9.91 & 0.19 & 99.14 & 9.97 & 0.79 & 99.56 \\
\hline & 20.00 & 19.76 & 0.17 & 98.80 & 19.92 & 0.86 & 99.43 \\
\hline & 5.00 & 4.97 & 0.14 & 99.49 & 5.01 & 1.14 & 100.66 \\
\hline \multirow[t]{3}{*}{13} & 10.00 & 9.89 & 0.18 & 98.90 & 9.94 & 0.78 & 99.78 \\
\hline & 20.00 & 19.73 & 0.20 & 98.64 & 19.88 & 0.84 & 99.57 \\
\hline & 5.00 & 4.98 & 0.21 & 99.65 & 5.03 & 1.42 & 100.70 \\
\hline 14 & 10.00 & 9.90 & 0.22 & 98.99 & 9.96 & 1.09 & 100.51 \\
\hline & 20.00 & 19.74 & 0.15 & 98.68 & 19.89 & 0.55 & 100.93 \\
\hline & 5.00 & 4.99 & 0.10 & 99.83 & 5.03 & 1.18 & 100.70 \\
\hline 15 & 10.00 & 9.92 & 0.16 & 99.20 & 9.98 & 0.92 & 99.89 \\
\hline & 20.00 & 19.76 & 0.20 & 98.78 & 19.91 & 1.00 & 99.63 \\
\hline & 5.00 & 4.97 & 0.28 & 99.47 & 5.04 & 1.17 & 100.55 \\
\hline 16 & 10.00 & 9.95 & 0.20 & 99.50 & 10.05 & 0.86 & 99.67 \\
\hline & 20.00 & 20.13 & 0.33 & 100.66 & 20.19 & 0.92 & 99.54 \\
\hline & 5.00 & 4.99 & 0.12 & 99.70 & 5.04 & 1.14 & 100.71 \\
\hline 17 & 10.00 & 9.90 & 0.18 & 99.04 & 9.99 & 0.87 & 100.09 \\
\hline & 20.00 & 19.74 & 0.21 & 98.72 & 19.93 & 0.96 & 99.79 \\
\hline & 5.00 & 4.98 & 0.12 & 99.63 & 5.03 & 1.07 & 99.22 \\
\hline 18 & 10.00 & 9.90 & 0.17 & 98.98 & 9.97 & 0.72 & 100.23 \\
\hline & 20.00 & 19.74 & 0.18 & 98.72 & 19.91 & 0.56 & 99.86 \\
\hline
\end{tabular}


Table 3. Cont.

\begin{tabular}{|c|c|c|c|c|c|c|c|}
\hline \multirow[b]{2}{*}{ Analyte } & \multirow[b]{2}{*}{$\begin{array}{l}\text { Conc. } \\
(\mu \mathrm{g} / \mathrm{mL})\end{array}$} & \multicolumn{3}{|c|}{ Intraday $(n=5)$} & \multicolumn{3}{|c|}{ Interday $(n=5)$} \\
\hline & & $\begin{array}{c}\text { Measured } \\
\text { Conc. } \\
(\mu \mathrm{g} / \mathrm{mL})\end{array}$ & $\begin{array}{l}\text { Precision } \\
\text { (RSD, \%) }^{a}\end{array}$ & Accuracy (\%) & $\begin{array}{c}\text { Measured } \\
\text { Conc. } \\
(\mu \mathrm{g} / \mathrm{mL})\end{array}$ & $\begin{array}{l}\text { Precision } \\
\text { (RSD, \%) }\end{array}$ & Accuracy (\%) \\
\hline \multirow{4}{*}{19} & 5.00 & 4.99 & 0.14 & 99.76 & 5.04 & 1.20 & 100.82 \\
\hline & 10.00 & 9.93 & 0.13 & 99.28 & 10.01 & 0.94 & 99.97 \\
\hline & 20.00 & 19.79 & 0.22 & 98.94 & 19.96 & 0.97 & 99.74 \\
\hline & 5.00 & 4.95 & 0.37 & 99.09 & 4.96 & 0.90 & 101.19 \\
\hline \multirow[t]{3}{*}{20} & 10.00 & 10.04 & 0.42 & 100.44 & 10.02 & 0.63 & 100.45 \\
\hline & 20.00 & 19.89 & 0.24 & 99.47 & 19.97 & 0.72 & 100.29 \\
\hline & 5.00 & 4.99 & 0.29 & 99.86 & 5.04 & 1.11 & 100.60 \\
\hline \multirow[t]{3}{*}{21} & 10.00 & 9.91 & 0.22 & 99.14 & 10.00 & 0.82 & 99.64 \\
\hline & 20.00 & 19.78 & 0.27 & 98.90 & 19.95 & 0.89 & 99.44 \\
\hline & 5.00 & 5.03 & 0.18 & 100.58 & 5.06 & 1.12 & 100.48 \\
\hline \multirow[t]{3}{*}{22} & 10.00 & 10.00 & 0.10 & 99.99 & 10.05 & 0.80 & 99.55 \\
\hline & 20.00 & 19.94 & 0.23 & 99.72 & 20.06 & 0.87 & 99.42 \\
\hline & 5.00 & 4.99 & 0.17 & 99.75 & 5.03 & 1.04 & 100.26 \\
\hline \multirow[t]{3}{*}{23} & 10.00 & 9.90 & 0.21 & 99.02 & 9.96 & 0.75 & 99.43 \\
\hline & 20.00 & 19.73 & 0.18 & 98.66 & 19.89 & 0.84 & 99.38 \\
\hline & 5.00 & 4.98 & 0.19 & 99.62 & 5.02 & 1.12 & 100.50 \\
\hline \multirow[t]{2}{*}{24} & 10.00 & 9.90 & 0.19 & 98.96 & 9.96 & 0.79 & 99.56 \\
\hline & 20.00 & 19.73 & 0.17 & 98.64 & 19.88 & 0.86 & 99.43 \\
\hline
\end{tabular}

${ }^{a} \operatorname{RSD}(\%)=$ standard deviation (SD) $/$ mean $\times 100 \%$.

\subsection{Simultaneous Determination of 24 Markers Components for Quality Assessment of DHGST Sample}

The established HPLC analysis method was successfully applied to the simultaneous determination of DHGST components for quality assessment. Simultaneous analysis of DHGST using the established assays showed that compounds 1-24 were present in $0.23-14.68 \mathrm{mg} / \mathrm{g}$ of freeze-dried sample (Table 4).

Table 4. Content of the 24 marker analytes in the DHGST sample determined using HPLC $(n=3)$.

\begin{tabular}{|c|c|c|c|c|c|c|c|}
\hline \multirow{3}{*}{ Analyte } & \multicolumn{6}{|c|}{ Content (mg/g Freeze-Dried Sample) } & \multirow{3}{*}{ Source ${ }^{a}$} \\
\hline & \multicolumn{2}{|c|}{ Batch 1} & \multicolumn{2}{|c|}{ Batch 2} & \multicolumn{2}{|c|}{ Batch 3} & \\
\hline & Mean & RSD (\%) & Mean & RSD (\%) & Mean & $\operatorname{RSD}\left(\%, \times 10^{-1}\right)$ & \\
\hline 1 & 0.70 & 0.20 & 0.71 & 0.28 & 0.70 & 0.02 & PL, TC \\
\hline 2 & 0.61 & 0.30 & 0.61 & 0.22 & 0.61 & 2.29 & RG \\
\hline 3 & 2.91 & 0.40 & 2.99 & 0.25 & 2.96 & 3.61 & EU \\
\hline 4 & 2.73 & 0.22 & 2.79 & 0.25 & 2.74 & 0.89 & GS \\
\hline 5 & 0.94 & 1.22 & 0.95 & 0.91 & 0.95 & 6.23 & EU \\
\hline 6 & 14.29 & 0.19 & 14.68 & 0.84 & 14.48 & 7.08 & GS \\
\hline 7 & 9.81 & 0.26 & 9.69 & 0.44 & 9.70 & 5.92 & EU \\
\hline 8 & 3.13 & 0.28 & 3.28 & 1.16 & 3.28 & 24.73 & PL \\
\hline 9 & 0.30 & 1.00 & 0.30 & 0.22 & 0.30 & 5.05 & SD \\
\hline 10 & 4.68 & 0.30 & 4.77 & 0.28 & 4.72 & 2.19 & PL \\
\hline 11 & 1.25 & 0.51 & 1.27 & 0.22 & 1.25 & 5.23 & GU \\
\hline 12 & 0.24 & 1.26 & 0.23 & 0.99 & 0.23 & 5.14 & GU \\
\hline 13 & 0.23 & 0.98 & 0.23 & 0.09 & 0.23 & 1.75 & $\mathrm{CO}$ \\
\hline 14 & 0.90 & 0.23 & 0.90 & 0.29 & 0.90 & 3.48 & AG \\
\hline 15 & 0.26 & 0.31 & 0.26 & 0.91 & 0.26 & 1.66 & SD \\
\hline 16 & 0.52 & 0.47 & 0.52 & 0.28 & 0.52 & 0.91 & PL \\
\hline 17 & 0.39 & 0.57 & 0.39 & 0.50 & 0.39 & 0.74 & CC \\
\hline 18 & 0.60 & 0.40 & 0.60 & 0.31 & 0.60 & 1.80 & CC \\
\hline 19 & 1.36 & 0.50 & 1.38 & 0.48 & 1.36 & 4.64 & CC \\
\hline 20 & 2.25 & 0.44 & 2.28 & 1.27 & 2.26 & 7.34 & GU \\
\hline
\end{tabular}


Table 4. Cont.

\begin{tabular}{|c|c|c|c|c|c|c|c|}
\hline \multirow{3}{*}{ Analyte } & \multicolumn{6}{|c|}{ Content (mg/g Freeze-Dried Sample) } & \multirow{3}{*}{ Source ${ }^{a}$} \\
\hline & \multicolumn{2}{|c|}{ Batch 1} & \multicolumn{2}{|c|}{ Batch 2} & \multicolumn{2}{|c|}{ Batch 3} & \\
\hline & Mean & RSD (\%) & Mean & RSD (\%) & Mean & $\operatorname{RSD}\left(\%, \times 10^{-1}\right)$ & \\
\hline 21 & 1.38 & 0.30 & 1.39 & 0.82 & 1.39 & 5.41 & $\mathrm{AH}$ \\
\hline 22 & 0.50 & 1.16 & 0.50 & 0.63 & 0.50 & 9.28 & $\mathrm{AH}$ \\
\hline 23 & 0.25 & 0.20 & 0.25 & 0.01 & 0.25 & 3.45 & AG \\
\hline 24 & 0.24 & 0.23 & 0.25 & 0.11 & 0.24 & 1.48 & AG \\
\hline
\end{tabular}

a PL, P. lactiflora; TC, T. chinensis; RG, R. glutinosa; EU, E. ulmoides; GS, G. straminea; SD, S. divaricate; GU, G. uralensis; CO, C. officinale; AG, A. gigas; CC, C. cassia; and AH, A. heterotropoides.

\section{Conclusions}

A simultaneous HPLC analysis method for quality assessment of DHGST, a traditional herbal medicine prescription that has been used for arthritis and neuritis for a long time, was developed. The method was validated with respect to linearity, LOD, LOQ, recovery, and precision. As a result of these investigations, components of G. straminea, E. ulmoides, and P. lactiflora in the DHGST sample, such as gentiopicroside $(6,14.29-14.68 \mathrm{mg} / \mathrm{g})$, pinoresinol diglucoside $(7,9.69-9.81 \mathrm{mg} / \mathrm{g})$, and paeoniflorin $(\mathbf{1 0}, 4.68-4.77 \mathrm{mg} / \mathrm{g})$, were found in the highest amounts. The developed and validated method is suitable for use in the quality assessment of DHGST or other herbal medicine prescriptions.

Supplementary Materials: The following are available online at https:/ /www.mdpi.com/article/ 10.3390/app11177829/s1, Figure S1: Chemical structures of the 24 marker components in DHGST; Figure S2: HPLC chromatogram of the herbal medicine extract and its main components. (A): A. continentalis; (B): A. gigas; (C), P. lactiflora; (D), T. chinensis; (E), R. glutinosa; (F), C. officinale; (G), P. ginseng; $(\mathrm{H})$, P. cocos; (I), A. bidentata; (J), E. ulmoides; (K), G. straminea; (L), A. heterotropoides; (M), S. divaricate; $(\mathrm{N})$, C. cassia; $(\mathrm{O})$, G. uralensis; and (P), Z. officinale; Figure S3: HPLC chromatograms of the standard solution (A) and 70\% methanol of DHGST sample (B). Gallic acid (1), 5-hydroxymethylfurfural (2), geniposidic acid (3), loganic acid (4), cimufugin (5), chlorogenic acid (6), geniposide (7), gentiopicroside (8), pinoresinol diglucoside (9), albiflorin (10), prim-O-glucosylcimifugin (11), paeoniflorin (12), genipin (13), liquiritin apioside (14), liquiritin (15), ecdysterone (16), ferulic acid (17), nodakenin (18), 5-O-methylvisammioside (19), quercitrin (20), benzoic acid (21), ginsenoside Rg1 (22), coumarin (23), cinnamic acid (24), benzoylpaeoniflorin (25), ginsenoside Rb1 (26), cinnamaldehyde (27), glycyrrhizin (28), 6-gingerol (29), aristolochic acid II (30), aristolochic acid I (31), $\beta$-asarone (32), methyleugenol (33), $\alpha$-asarone (34), safrole (35), decursin (36), decursinol angelate (37), pachymic acid (38), continentalic acid (39), and kaurenoic acid (40); Table S1: Information and composition of DHGST; Table S2: HPLC operating conditions for simultaneous quantification of the 24 marker components in DHGST; Table S3: System suitability for the analysis of the 24 marker compounds with the developed HPLC method; Table S4: Repeatability of retention time of the 24 marker analytes using HPLC $(n=6)$; Table S5: Repeatability of peak area of the 24 marker analytes by HPLC $(n=6)$.

Author Contributions: Conceptualization, C.-S.S. and H.-K.S.; performing experiments, analyzing data, and writing — original draft preparation, C.-S.S.; funding acquisition, H.-K.S. All authors have read and agreed to the published version of the manuscript.

Funding: This research was supported by grants from the Korea Institute of Oriental Medicine (grant numbers KSN2013310 and KSN2021310).

Institutional Review Board Statement: Not applicable.

Informed Consent Statement: Not applicable.

Conflicts of Interest: The authors have declared no conflict of interest.

\section{References}

1. Heo, J. Donguibogam; Namsandang: Seoul, Korea, 2007; p. 303.

2. Xiong, Z.; Zheng, C.; Chang, Y.; Liu, K.; Shu, L.; Zhang, C. Exploring the pharmacological mechanism of Duhuo Jisheng decoction in treating osteoporosis based on network pharmacology. Evid.-Based Complement. Alternat. Med. 2021, 2021, 5510290. [CrossRef] 
3. Zhou, X.; Xiang, K.; Lu, M.; Xia, H.; Zhou, X.; Yuan, X.; Wang, Z.; Li, K. A comparative study of the efficacy of Chinese herbal medicine Duhuo Jisheng decoction combined with DMARDs vs isolated DMARDs for rheumatoid arthritis. Medicine 2020, 99, e23479. [CrossRef]

4. Sun, K.; Huang, F.; Qi, B.; Yin, H.; Tang, B.; Yang, B.; Chen, L.; Zhuang, M.; Wei, X.; Zhu, L. A systematic review and meta-analysis for Chinese herbal medicine Duhuo Jisheng decoction in treatment of lumbar disc herniation: A protocol for a systematic review. Medicine 2020, 99, e19310. [CrossRef]

5. Xiong, Z.; Yi, P.; Zhang, L.; Ma, H.; Li, W.; Tan, M. Efficacy and safety of modified Duhuo Jisheng decection in the treatment of lumbar disc herniation: A systematic review and meta-analysis. Evid.-Based Complement. Alternat. Med. 2020, $2020,2381462$. [CrossRef]

6. Li, J.; Wang, W.; Feng, G.; Du, J.; Kang, S.; Li, Z.; Zhu, W.; Shang, H. Efficacy and safety of Duhuo Jisheng decoction for postmenopausal osteoporosis: A systematic review and meta-analysis. Evid.-Based Complement. Alternat. Med. 2020, 2020, 6957825. [CrossRef]

7. Zhang, W.; Wang, S.; Zhang, R.; Zhang, Y.; Li, X.; Lin, Y.; Wei, X. Evidence of Chinese herbal medicine Duhuo Jisheng decoction for knee osteoarthritis: A systematic review of randomized clinical trials. BMJ Open 2016, 6, e008973. [CrossRef] [PubMed]

8. Liu, Z.; Wang, Z.; Huang, C.; Fu, Z.; Liu, Y.; Wei, Z.; Liu, S.; Ma, C.; Shen, J.; Duan, D.D. Duhuo Jisheng decoction inhibits SDF-1induced inflammation and matrix degradation in human degenerative nucleus pulposus cells in vitro through the CXCR4/NF- $\mathrm{KB}$ pathway. Acta Pharmacol. Sin. 2018, 39, 912-922. [CrossRef]

9. Liu, W.; Jin, S.; Huang, M.; Li, Y.; Wang, Z.W.; Wang, P.; Zhao, X.; Xia, P.; Feng, J. Duhuo jisheng decoction suppresses matrix degradation and apoptosis in human nucleus pulposus cells and ameliorates disc degeneration in a rat model. J. Ethnopharmacol. 2020, 250, 112494. [CrossRef]

10. Kim, H.S.; Moon, B.C.; Choi, G.; Kim, W.J.; Lee, A.Y. Ultra-performance convergence chromatography for the quantitative determination of bioactive compounds in Aralia continentalis Kitagawa as quality control markers. J. Sep. Sci. 2017, 40, 2071-2079. [CrossRef]

11. Ahn, M.J.; Lee, M.K.; Kim, Y.C.; Sung, S.H. The simultaneous determination of coumarins in Angelica gigas root by high performance liquid chromatography-diode array detector coupled with electrospray ionization/mass spectrometry. J. Pharm. Biomed. Anal. 2008, 46, 258-266. [CrossRef]

12. Bae, J.Y.; Kim, C.Y.; Kim, H.J.; Park, J.H.; Ahn, M.J. Differences in chemical profiles and biological activities of Paeonia lactiflora and Paeonia obovata. J. Med. Food 2015, 18, 224-232. [CrossRef] [PubMed]

13. Liu, R.; Su, B.; Huang, F.; Ru, M.; Zhang, H.; Qin, Z.; Li, Y.; Zhu, K. Identification and analysis of cardiac glycosides in Loranthaceae parasites Taxillus chinensis (DC.) Danser and Scurrula parasitica Linn. and their host Nerium indicum Mill. J. Pharm. Biomed. Anal. 2019, 174, 450-459. [CrossRef]

14. Lee, J.Y.; Lee, E.J.; Kim, J.S.; Lee, J.H.; Kang, S.S. Phytochemical studies on Rehmanniae Radix Preparata. Kor. J. Pharmacogn. 2011, $42,117-126$

15. Baek, M.E.; Seong, G.U.; Lee, Y.J.; Won, J.H. Quantitative analysis for the quality evaluation of active ingredients in Cnidium Rhizome. Yakhak Hoeji 2016, 60, 227-234. [CrossRef]

16. Shan, S.M.; Luo, J.G.; Huang, F.; Kong, L.Y. Chemical characteristics combined with bioactivity for comprehensive evaluation of Panax ginseng C.A. Meyer in different ages and seasons based on HPLC-DAD and chemometric methods. J. Pharm. Biomed. Anal. 2014, 89, 76-82. [CrossRef]

17. Li, G.; Xu, M.L.; Lee, J.S.; Woo, M.H.; Chang, H.W.; Son, J.K. Cytotoxicity and DNA topoisomerases inhibitory activity of constituents from the Sclerotium of Poria cocos. Arch. Pharm. Res. 2004, 27, 829-833. [CrossRef]

18. Zhao, B.T.; Jeong, S.Y.; Moon, D.C.; Son, K.H.; Son, J.K.; Woo, M.H. High performance liquid chromatography used for quality control of Achyranthis Radix. Arch. Pharm. Res. 2012, 35, 1449-1455. [CrossRef]

19. Zhao, B.T.; Jeong, S.Y.; Kim, T.I.; Seo, E.K.; Min, B.S.; Son, J.K.; Woo, M.H. Simultaneous quantitation and validation of method for the quality evaluation of Eucommiae Cortex by HPLC/UV. Arch. Pharm. Res. 2015, 38, 2183-2192. [CrossRef]

20. Liu, F.F.; Wang, Y.M.; Zhu, H.T.; Wang, D.; Yang, C.R.; Xu, M.; Zhang, Y.J. Comparative study on "Long-Dan”, "Qin-Jiao" and their adulterants by HPLC analysis. Nat. Prod. Bioprospect. 2014, 4, 297-308. [CrossRef]

21. Seo, C.S.; Shin, H.K. Quantitative analysis of the seven marker components in Asarum sieboldii using the LC-MS/MS and GC-MS. Kor. J. Pharmacogn. 2013, 44, 350-361.

22. Kim, M.K.; Yang, D.H.; Jung, M.; Jung, E.H.; Eom, H.Y.; Suh, J.H.; Min, J.W.; Kim, U.; Min, H.; Kim, J.; et al. Simultaneous determination of chromones and coumarin in Radix Saposhnikoviae by high performance liquid chromatography with diode array and tandem mass detectors. J. Chromatogr. A 2011, 1218, 6319-6330. [CrossRef]

23. Lv, G.P.; Huang, W.H.; Yang, F.Q.; Li, J.; Li, S.P. Pressurized liquid extraction and GC-MS analysis for simultaneous determination of seven components in Cinnamomum cassia and the effect of sample preparation. J. Sep. Sci. 2010, 33, 2341-2348. [CrossRef] [PubMed]

24. Zhou, S.; Cao, J.; Qiu, F.; Kong, W.; Yang, S.; Yang, M. Simultaneous determination of five bioactive components in Radix Glycyrrhizae by pressurised liquid extraction combined with UPLC-PDA and UPLC/ESI-QTOF-MS confirmation. Phytochem. Anal. 2013, 24, 527-533. [CrossRef] [PubMed] 
25. Lee, H.R.; Lee, J.H.; Park, C.S.; Ra, K.R.; Ha, J.S.; Cha, M.H.; Kim, S.N.; Choi, Y.; Hwang, J.; Nam, J.S. Physicochemical properties and antioxidant capacities of different parts of ginger (Zingiber officinale Roscoe). J. Korean Soc. Food Sci. Nutr. 2014, 43, 1369-1379. [CrossRef]

26. Chen, Y.; Li, J.; Li, Q.; Wang, T.; Xing, L.; Xu, H.; Wang, Y.; Shi, Q.; Zhou, Q.; Liang, Q. Du-Huo-Ji-Sheng-Tang attenuates inflammation of TNF-Tg Mice related to promoting lymphatic drainage function. Evid.-Based Complement. Alternat. Med. 2016, 2016, 7067691. [CrossRef]

27. Wang, J.Y.; Chen, W.M.; Wen, C.S.; Huang, S.C.; Chen, P.W.; Chiu, J.H. Du-Huo-Ji-Sheng-Tang and its active component ligusticum chuanxiong promote osteogenic differentiation and decrease the aging process of human mesenchymal stem cells. $J$. Ethnopharmacol. 2017, 198, 64-72. [CrossRef]

28. Lee, K.H. The Dispensatory on the Visual and Organoleptic Examination of Herbal Medicine; National Institute of Food and Drug Safety Evaluation: Seoul, Korea, 2013; pp. 24-599.

29. Seo, C.S.; Lee, M.Y. Simultaneous quantification of eight marker components in traditional herbal formula, Haepyoyijin-tang using HPLC-PDA. Appl. Sci. 2020, 10, 3888. [CrossRef]

30. Seo, C.S.; Shin, H.K. Quantitative analysis of 18 marker components in the traditional Korean medicine, Cheongsangbangpungtang, using high-performance liquid chromatography combined with photodiode array detector. Appl. Sci. 2021, 11, 14. [CrossRef] 